\title{
LONG PATHS IN RANDOM APOLLONIAN NETWORKS
}

\author{
Colin Cooper ${ }^{1}$ and Alan Frieze ${ }^{2}$ \\ ${ }^{I}$ Department of Informatics, King's College, University of London, London, UK \\ ${ }^{2}$ Department of Mathematical Sciences, Carnegie Mellon University, Pittsburgh, \\ Pennsylvania, USA
}

\begin{abstract}
We consider the length $L(n)$ of the longest path in a randomly generated Apollonian Network (ApN) $\mathcal{A}_{n}$. We show that with high probability $L(n) \leq n e^{-\log ^{c} n}$ for any constant $c<2 / 3$.
\end{abstract}

\section{INTRODUCTION}

This article concerns the length of the longest path in a random Apollonian Network (ApN) $\mathcal{A}_{n}$. We start with a triangle $T_{0}=x y z$ in the plane. We then place a point $v_{1}$ in the centre of this triangle, creating three triangular faces. We choose one of these faces at random and place a point $v_{2}$ in its middle. There are now five triangular faces. We choose one at random and place a point $v_{3}$ in its center. In general, after we have added $v_{1}, v_{2}, \ldots, v_{1}$, there will be $2 n+1$ triangular faces. We choose one at random and place $v_{n}$ inside it. The random graph $\mathcal{A}_{n}$ is the graph induced by this embedding. It has $n+3$ vertices and $3 n+6$ edges.

This graph has been the object of study recently; in the context of scale-free graphs [5]. Properties of its degree sequence, properties of the spectra of its adjacency matrix, and its diameter were determined. The diameter result was improved, and the diameter was determined asymptotically $[3,4]$. The study [4] proves the following result concerning the length of the longest path in $\mathcal{A}_{n}$ :

Theorem 1.1. There exists an absolute constant $\alpha$ such that if $L(n)$ denotes the length of the longest path in $\mathcal{A}_{n}$ then

$$
\operatorname{Pr}\left(L(n) \geq \frac{n}{\log ^{\alpha} n}\right) \leq \frac{1}{\log ^{\alpha} n} .
$$

Address correspondence to Alan Frieze, Department of Mathematical Sciences, Carnegie Mellon University, Pittsburgh, PA 15213, USA. E-mail: alan@random.math.mu.edu 
The value of $\alpha$ from [4] is rather small, and we will assume for the purposes of this proof that

$$
\alpha<\frac{1}{3}
$$

The aim of this work is to give the following improvement on Theorem 1.1:

\section{Theorem 1.2.}

$$
\operatorname{Pr}\left(L(n) \geq n e^{-\log ^{c} n}\right) \leq O\left(e^{-\log ^{c / 2} n}\right)
$$

for any constant $c<2 / 3$.

This is most likely far from the truth. It is reasonable to conjecture that, in fact, $L(n) \leq n^{1-\varepsilon}$ w.h.p. for some positive $\varepsilon>0$. For lower bounds, [4] shows that $L(n) \geq n^{\log _{3} 2}+2$ always and $\mathbf{E}(L(n))=\Omega\left(n^{0.8}\right)$. An $\Omega\left(n^{\log _{3} 2}\right)$ lower bound for arbitrary 3-connected planar graphs has been proven [1].

\section{OUTLINE PROOF STRATEGY}

We take an arbitrary path $P$ in $\mathcal{A}_{n}$ and bound its length as follows. We add vertices to the interior of $x y z$ in rounds. In round $i$ we add $\sigma_{i}$ vertices. We start with $\sigma_{0}=n^{1 / 2}$ and choose $\sigma_{i} \gg \sigma_{i-1}$, where $A \gg B$ i.f.f $B=o(A)$. We will argue inductively that $P$ visits only $\tau_{i-1}=o\left(\sigma_{i-1}\right)$ faces of $\mathcal{A}_{\sigma_{i-1}}$ and then use Lemma 4.1 to argue that roughly a fraction $\tau_{i-1} / \sigma_{i-1}$ of the $\sigma_{i}$ new vertices go into faces visited by $P$. We then use a variant (Lemma 5.1) of Theorem 1.1 to argue that w.h.p. $\frac{\tau_{i}}{\sigma_{i}} \leq \frac{\tau_{i-1}}{2 \sigma_{i-1}}$. Theorem 1.2 will follow easily from this.

\section{PATHS AND TRIANGLES}

Fix $1 \leq \sigma \leq n$ and let $\mathcal{A}_{\sigma}$ denote the ApN we have after inserting $\sigma$ vertices $A$ interior to $T_{0}$. It has $2 \sigma+1$ faces, which we denote by $\mathcal{T}=\left\{T_{1}, T_{2}, \ldots, T_{2 \sigma+1}\right\}$. Now add $N$ more vertices $B$ to create a larger network $\mathcal{A}_{\sigma^{\prime}}$, where $\sigma^{\prime}=\sigma+N$. Now consider a path $P=x_{1}, x_{2}, \ldots, x_{m}$ through $\mathcal{A}_{\sigma^{\prime}}$. Let $I=\left\{i: x_{i} \in A\right\}=\left\{i_{1}, i_{2}, \ldots, i_{\tau}\right\}$. Note that $Q=\left(i_{1}, i_{2}, \ldots, i_{\tau}\right)$ is a path of length $\tau-1$ in $\mathcal{A}_{\sigma}$. This is because $i_{k} i_{k+1}, 1 \leq k<\tau$ must be an edge of some face in $\mathcal{T}$. We also see that for any $1 \leq k<\tau$, the vertices $x_{j}, i_{k}<j<i_{k+1}$ will all be interior to the same face $T_{l}$ for some $l \in[2 \sigma+1]$.

We summarize this in the following lemma, using the notation of the preceding paragraph.

Lemma 3.1. Suppose that $1 \leq \sigma<\sigma^{\prime} \leq n$ and that $Q$ is a path of $\mathcal{A}_{\sigma}$ that is obtained from a path $P$ in $\mathcal{A}_{\sigma^{\prime}}$ by omitting the vertices in $B$. 
Suppose that $Q$ has $\tau$ vertices and that $P$ visits the interior of $\tau^{\prime}$ faces from $\mathcal{T}$. Then

$$
\tau-1 \leq \tau^{\prime} \leq \tau+1
$$

Proof. The path $P$ breaks into vertices of $\mathcal{A}_{\sigma}$ plus $\tau+1$ intervals where, in an interval, it visits the interior of a single face in $\mathcal{T}$. This justifies the upper bound. The lower bound comes from the fact that except for the face in which it starts, if $P$ reenters a face $x y z$, then it cannot leave it, because it will have already visited all three vertices $x, y, z$. Thus, at most two of the aforementioned intervals can represent a repeated face.

\section{A STRUCTURAL LEMMA}

Let

$$
\lambda_{1}=\log ^{2} n
$$

A sequence of events $\mathcal{E}_{n}$ holds quite surely (q.s.) if $\operatorname{Pr}\left(\neg \mathcal{E}_{n}\right)=O\left(n^{-K}\right)$ for any constant $K>0$.

Lemma 4.1. The following holds for all $i$. Let $\sigma=\sigma_{i}$ and suppose that $\lambda_{1} \leq \tau \ll \sigma$. Suppose that $T_{1}, T_{2}, \ldots, T_{\tau}$ is a set of triangularfaces of $\mathcal{A}_{\sigma}$. Suppose that $N \gg \sigma$ and that when adding $N$ vertices to $\mathcal{A}_{\sigma}$ we find that $M_{j}$ vertices are placed in $T_{j}$ for $j=1,2, \ldots, \tau$. Then, for all $J \subseteq[2 \sigma+1],|J|=\tau$, we have

$$
\sum_{j \in J} M_{j} \leq \frac{100 \tau N}{\sigma} \log \left(\frac{\sigma}{\tau}\right) .
$$

This holds q.s. for all choices of $\tau, \sigma$, and $T_{1}, T_{2}, \ldots, T_{\tau}$.

Proof. We consider the following process that starts with $s$ newly born particles. Once a particle is born, it waits an exponentially mean one distributed amount of time. After this time, it simultaneously dies and gives birth to $k$ new particles, and so on. A birth corresponds to a vertex of our network and a particle corresponds to a face.

Let $Z_{t}$ denote the number of deaths up to time $t$. The number of particles in the system is $\beta_{N}=s+N(k-1)$. Then we have

$$
\operatorname{Pr}\left(Z_{t+d t}=N\right)=\beta_{N-1} \operatorname{Pr}\left(Z_{t}=N-1\right) d t+\left(1-\beta_{N} d t\right) \operatorname{Pr}\left(Z_{t}=N\right) .
$$

So, if $p_{N}(t)=\operatorname{Pr}\left(Z_{t}=N\right)$, we have $f_{N}(0)=1_{N=s}$ and

$$
p_{N}^{\prime}(t)=\beta_{N-1} p_{N-1}(t)-\beta_{N} p_{N}(t)
$$


This yields

$$
\begin{aligned}
p_{N}(t) & =\prod_{i=1}^{N} \frac{(k-1)(i-1)+s}{(k-1) i} \times e^{-s t}\left(1-e^{-(k-1) t}\right)^{N} \\
& =A_{k, N, s} e^{-s t}\left(1-e^{-(k-1) t}\right)^{N} .
\end{aligned}
$$

We have $A_{3,0, s}=1$. When $s$ is even, $s, N \rightarrow \infty$, and $k=3$, we have

$$
\begin{aligned}
A_{3, N, s} & =\prod_{i=1}^{N}\left(\frac{s / 2+i-1}{i}\right)=\left(\begin{array}{c}
N+s / 2-1 \\
s / 2-1
\end{array}\right) \\
& \approx\left(1+\frac{s-2}{2 N}\right)^{N}\left(1+\frac{2 N}{s-2}\right)^{s / 2-1} \sqrt{\frac{2 N+s}{2 \pi N s}} .
\end{aligned}
$$

We also need to have an upper bound for a small, even $s, N^{2}=o(s)$, say. In this case, we use

$$
A_{3, N, s} \leq s^{N}
$$

When $s \geq 3$ is odd, $s, N \rightarrow \infty$ (no need to deal with small $N$ here), and $k=3$ we have

$$
\begin{aligned}
A_{3, N, s} & =\prod_{i=1}^{N}\left(\frac{2 i-2+s}{2 i}\right)=\frac{(s-1+2 N) !((s-1) / 2) !}{2^{2 N}(s-1) ! N !((s-1) / 2+N) !} \\
& \approx\left(1+\frac{s-1}{2 N}\right)^{N}\left(1+\frac{2 N}{s-1}\right)^{(s-1) / 2} \frac{1}{(2 \pi N)^{1 / 2}} .
\end{aligned}
$$

We now consider $\tau \rightarrow \infty, \tau \ll \sigma, N \geq m \geq 2 \tau N / \sigma \gg \tau$ and arbitrary $t$ (under the assumption that $\tau$ is odd and $\sigma$ is odd). (We sometimes use $A \leq_{b} B$ in place of $A=O(B)$ ).

$$
\begin{aligned}
& \operatorname{Pr}\left(M_{1}+\cdots+M_{\tau}=m \mid M_{1}+\cdots+M_{\sigma}=N\right) \\
& =\frac{\operatorname{Pr}\left(M_{1}+\cdots+M_{\tau}=m\right) \operatorname{Pr}\left(M_{\tau+1}+\cdots+M_{\sigma}=N-m\right)}{\operatorname{Pr}\left(M_{1}+\cdots+M_{\sigma}=N\right)} \\
& =\frac{A_{3, m, \tau} A_{3, N-m, \sigma-\tau}}{A_{3, N, \sigma}} \\
& \approx \frac{\left(1+\frac{\tau-1}{2 m}\right)^{m}\left(1+\frac{2 m}{\tau-1}\right)^{(\tau-1) / 2}\left(1+\frac{\sigma-\tau-2}{2(N-m)}\right)^{N-m}\left(1+\frac{2(N-m)}{\sigma-\tau-2}\right)^{(\sigma-\tau-2) / 2}(N(2(N-m)+\sigma))^{1 / 2}}{\left(1+\frac{\sigma-1}{2 N}\right)^{N}\left(1+\frac{2 N}{\sigma-1}\right)^{(\sigma-1) / 2}(2 \pi m \sigma(N-m))^{1 / 2}} \\
& \quad \leq_{b} \frac{e^{(\tau-1) / 2}\left(\left(\frac{2 m}{\tau}\right)^{(\tau-1) / 2} e^{o(\tau)}\right) e^{(\sigma-\tau) / 2}\left(1+\frac{2(N-m)}{\sigma-\tau-2}\right)^{(\sigma-\tau-2) / 2}(N(2(N-m)+\sigma))^{1 / 2}}{e^{\sigma / 2-\sigma^{2} / 8 N}\left(\left(\frac{2 N}{\sigma}\right)^{(\sigma-1) / 2} e^{\sigma^{2} /(4+o(1)) N}\right)(m \sigma(N-m))^{1 / 2}}
\end{aligned}
$$




$$
\leq_{b} \frac{e^{o(\tau)}\left(\frac{2 m}{\tau}\right)^{(\tau-1) / 2}\left(1+\frac{2(N-m)}{\sigma-\tau-2}\right)^{(\sigma-\tau-2) / 2}(N(2(N-m)+\sigma))^{1 / 2}}{\left(\frac{2 N}{\sigma}\right)^{(\sigma-1) / 2}(m \sigma(N-m))^{1 / 2}}
$$

The above bound can be rewritten as

$$
\leq_{b} \frac{e^{o(\tau)}\left(\frac{2}{\tau}\right)^{(\tau-1) / 2} N^{1 / 2} \sigma^{(\sigma-1) / 2}}{(2 N)^{(\sigma-1) / 2} \sigma^{1 / 2}} \times \frac{m^{(\tau-1) / 2}\left(1+\frac{2(N-m)}{\sigma-\tau-2}\right)^{(\sigma-\tau-2) / 2}(N-m+\sigma)^{1 / 2}}{(m(N-m))^{1 / 2}} .
$$

Suppose first that $m \leq N-4 \sigma$. Then, the bound becomes

$$
\begin{aligned}
& \leq_{b} \frac{e^{o(\tau)}\left(\frac{2}{\tau}\right)^{(\tau-1) / 2} N^{1 / 2} \sigma^{(\sigma-1) / 2}}{(2 N)^{(\sigma-1) / 2} \sigma^{1 / 2}} \times m^{(\tau-2) / 2}\left(1+\frac{2(N-m)}{\sigma-\tau-2}\right)^{(\sigma-\tau-2) / 2} \\
& \leq_{b} \frac{e^{o(\tau)} 2^{(\tau-1) / 2} N^{1 / 2} \sigma^{(\sigma-1) / 2}}{(2 N)^{(\sigma-1) / 2} \tau^{\tau / 2}} \times m^{(\tau-2) / 2}\left(\frac{2(N-m)}{\sigma-\tau}\right)^{(\sigma-\tau) / 2} e^{\sigma^{2} /(N-m)} \\
& \leq \frac{e^{o(\tau)} N^{1 / 2}}{m^{1 / 2}}\left(\frac{\sigma(N-m)}{N(\sigma-\tau)}\right)^{(\sigma-\tau) / 2}\left(\frac{\sigma m}{\tau N}\right)^{(\tau-1) / 2} e^{\sigma^{2} /(N-m)} \\
& \leq_{b} \frac{e^{o(\tau)} N^{1 / 2}}{m^{1 / 2}}\left(\frac{e^{2} m \sigma}{\tau N} \cdot \exp \left\{-\frac{m(\sigma-\tau)}{(\tau-1) N}+\frac{2 \sigma^{2}}{(\tau-1)(N-m)}\right\}\right)^{(\tau-1) / 2} \\
& =\frac{e^{o(\tau)} N^{1 / 2}}{m^{1 / 2}}\left(\frac{e^{2} m \sigma}{\tau N} \cdot \exp \left\{-\frac{m \sigma}{(\tau-1) N}\left(1-\frac{\tau}{\sigma}-\frac{2 \sigma}{m}-\frac{2 \sigma}{N-m}\right)\right\}\right)^{(\tau-1) / 2} \\
& \leq \frac{e^{o(\tau)} N^{1 / 2}}{m^{1 / 2}}\left(\frac{e^{2} m \sigma}{\tau N} \cdot \exp \left\{-\frac{m \sigma}{3 \tau N}\right\}\right)^{(\tau-1) / 2} \cdot
\end{aligned}
$$

We inflate this by $n^{2}\left(\begin{array}{c}2 \sigma+1 \\ \tau\end{array}\right)$ to account for our choices for $\sigma, \tau, T_{1}, \ldots, T_{\tau}$ to get

$$
\leq_{b} n^{2} \frac{e^{o(\tau)} N^{1 / 2}}{m^{1 / 2}}\left(\frac{4 e^{4} m \sigma^{3}}{\tau^{3} N} \cdot \exp \left\{-\frac{m \sigma}{3 \tau N}\right\}\right)^{(\tau-1) / 2} .
$$

So, if $m_{0}=\frac{100 \tau N \log (\sigma / \tau)}{\sigma}$, then

$$
\begin{aligned}
& \sum_{m=m_{0}}^{N-4 \sigma} \operatorname{Pr}\left(\exists \sigma, \tau, T_{1}, \ldots, T_{\tau}: M_{1}+\cdots+M_{\tau}=m \mid M_{1}+\cdots+M_{\sigma}=N\right) \\
& \quad \leq{ }_{b} n^{2} e^{o(\tau)} N^{5 / 2} \sum_{m=m_{0}}^{N-4 \sigma}\left(\frac{4 e^{4} m \sigma^{3}}{\tau^{3} N} \cdot \exp \left\{-\frac{m \sigma}{3 \tau N}\right\}\right)^{(\tau-1) / 2} \\
& \quad \leq n^{2} e^{o(\tau)} N^{7 / 2}\left(\frac{4 e^{4} m_{0} \sigma^{3}}{\tau^{3} N} \cdot \exp \left\{-\frac{m_{0} \sigma}{3 \tau N}\right\}\right)^{(\tau-1) / 2},
\end{aligned}
$$


since $x e^{-A x}$ is decreasing for $A x \geq 1$,

$$
\begin{aligned}
& =n^{2} e^{o(\tau)} N^{7 / 2}\left(\frac{4 e^{4} m_{0} \sigma}{\tau N} \exp \left\{-\frac{m_{0} \sigma}{6 \tau N}\right\} \times \frac{\sigma^{2}}{\tau^{2}} \exp \left\{-\frac{m_{0} \sigma}{6 \tau N}\right\}\right)^{(\tau-1) / 2} \\
& \leq n^{2} N^{7 / 2}\left(400 e^{4+o(1)} \log \left(\frac{\sigma}{\tau}\right) \times e^{-50 / 3} \times \frac{\sigma^{2}}{\tau^{2}}\left(\frac{\tau}{\sigma}\right)^{50 / 3}\right)^{(\tau-1) / 2} \\
& =O\left(n^{-K}\right),
\end{aligned}
$$

for any constant $K>0$.

Suppose now that $N-4 \sigma \leq m \leq N-\sigma^{1 / 3}$. Then we can bound (4.2) by

$$
\begin{aligned}
& \leq_{b} \frac{e^{o(\tau)}\left(\frac{2}{\tau}\right)^{(\tau-1) / 2} \sigma^{(\sigma-1) / 2}}{(2 N)^{(\sigma-1) / 2}} \times m^{(\tau-1) / 2} e^{4 \sigma} \\
& \leq\left(\frac{e^{8} \sigma}{2 N}\right)^{(\sigma-\tau) / 2}\left(\frac{e^{8} \sigma}{\tau}\right)^{(\tau-1) / 2} .
\end{aligned}
$$

We inflate this by $n^{2}\left(\begin{array}{c}2 \sigma+1 \\ \tau\end{array}\right)<n^{2} 4^{\sigma}$ to get

$$
\leq_{b} n^{2}\left(\frac{8 e^{8} \sigma}{N}\right)^{(\sigma-\tau) / 2}\left(\frac{16 e^{8} \sigma}{\tau}\right)^{(\tau-1) / 2} .
$$

So,

$$
\begin{gathered}
\sum_{m=N-4 \sigma}^{N-\sigma^{1 / 3}} \operatorname{Pr}\left(\exists \sigma, \tau, T_{1}, \ldots, T_{\sigma}: M_{1}+\cdots+M_{\tau}=m \mid M_{1}+\cdots+M_{\sigma}=N\right) \\
\quad \leq{ }_{b} n^{2} N^{2} \sigma\left(\frac{8 e^{8} \sigma}{N}\right)^{(\sigma-\tau) / 2}\left(\frac{16 e^{8} \sigma}{\tau}\right)^{(\tau-1) / 2}=O\left(n^{-K}\right),
\end{gathered}
$$

for any constant $K>0$, since $\sigma \log N \gg \tau \log \sigma$.

When $m \geq N-\sigma^{1 / 3}$ we replace (4.1) by

$$
\begin{aligned}
& \leq_{b} \frac{\left(1+\frac{\tau-1}{2 m}\right)^{m}\left(1+\frac{2 m}{\tau-1}\right)^{(\tau-1) / 2} \sigma^{N-m} N^{1 / 2}}{\left(1+\frac{\sigma-1}{2 N}\right)^{N}\left(1+\frac{2 N}{\sigma-1}\right)^{(\sigma-1) / 2}(m \sigma)^{1 / 2}} \\
& \leq_{b} \frac{e^{\tau / 2+o(\tau)}\left(\frac{2 m}{\tau}\right)^{(\tau-1) / 2} \sigma^{N-m} N^{1 / 2}}{e^{\sigma}\left(\frac{2 N}{\sigma}\right)^{(\sigma-1) / 2} m^{1 / 2}} \\
& \leq_{b}\left(\frac{e^{1+o(1)} \sigma}{\tau}\right)^{(\tau-1) / 2}\left(\frac{\sigma}{2 N}\right)^{(\sigma-\tau) / 2} \sigma^{\sigma^{1 / 3}}
\end{aligned}
$$

Inflating this by $n^{2} 4^{\sigma}$ gives a bound of

$$
\leq_{b} n^{2}\left(\frac{16 e^{1+o(1)} \sigma}{\tau}\right)^{(\tau-1) / 2}\left(\frac{8 \sigma^{1+o(1)}}{N}\right)^{(\sigma-\tau) / 2}=O\left(n^{- \text {any constant }}\right) .
$$




\section{MODIFICATIONS OF THEOREM 1.1}

Let $\lambda=\log ^{3} n$ and partition [ $\left.\lambda\right]$ into $q=\log n$ sets of size $\lambda_{1}=\log ^{2} n$. Now add $n-\lambda$ vertices to $\mathcal{T}_{\lambda}$ and let $M_{i}$ denote the number of vertices that land in the $i$ th part $\Pi_{i}$ of the partition. Lemma 4.1 implies that q.s.

$$
M_{i} \leq M_{\max }=\frac{200 n}{\log n} \log \log n, \quad 1 \leq i \leq \tau .
$$

Let

$$
\omega_{1}(x)=\log ^{\alpha / 2} x
$$

for $x \in \mathbb{R}$.

Let $L_{i}$ denote the length of the longest path in $\Pi_{i}$. Suppose that $\mathcal{T}_{n}$ contains a path of length at least $n / \omega_{1}, \omega_{1}=\omega_{1}(n)$ and let $k$ be the number of $i$ such that

$$
L_{i} \geq \frac{200 n \log \log n}{\omega_{1}^{2} \log n} \geq \frac{M_{\max }}{\log ^{\alpha}\left(M_{\max }\right)} .
$$

Then, as $k \leq q=\log n$ we have

$$
k \frac{200 n \log \log n}{\log n}+(\log n-k) \frac{200 n \log \log n}{\omega_{1}^{2} \log n} \geq \frac{n}{\omega_{1}},
$$

which implies that

$$
k \geq \frac{\log n}{201 \omega_{1} \log \log n} .
$$

Theorem 1.1 with the bound on $M_{i}$ given in (5.1) implies that the probability of this is at most

$$
\frac{1}{n}+\left(\begin{array}{c}
\log n \\
\frac{\log n}{201 \omega_{1} \log \log n}
\end{array}\right)\left(\frac{1}{\log ^{\alpha}(n / \log n)}\right)^{\frac{\log n}{201 \omega_{1} \log \log n}} \leq \frac{1}{n}+\left(\frac{1}{\log ^{\alpha / 3} n}\right)^{\frac{\log n}{201 \omega_{1} \log \log n}} \leq \frac{1}{\phi\left(n, \omega_{1}\right)}
$$

where

$$
\phi(x, y)=\exp \left\{\frac{\log x}{y \log \log x}\right\} .
$$

The term $1 / n$ accounts for the failure of the property in Lemma 4.1.

In summary, we have proved the following:

\section{Lemma 5.1.}

$$
\operatorname{Pr}\left(L(n) \geq \frac{n}{\omega_{1}(n)}\right) \leq \frac{1}{\phi\left(n, \omega_{1}\right)}
$$


We are using $\phi(x, y)$ in place of $\phi(x)$ because we will need to use $\omega_{1}(x)$ for values of $x$ other than $n$.

Next consider $\mathcal{A}_{\sigma}$ and $\lambda_{1} \leq \tau \ll \sigma$ and let $T_{1}, T_{2}, \ldots, T_{\tau}$ be a set of $\tau$ triangular faces of $\mathcal{A}_{\sigma}$. Suppose that we add $N \gg \sigma$ more vertices and let $N_{j}$ be the number of vertices that are placed in $T_{j}, 1 \leq j \leq \tau$.

Next let

$$
\Lambda(x)=e^{x^{2}}
$$

where $x \in \mathbb{R}$.

Now let

$$
J=\left\{j: N_{j} \geq \Lambda_{0}\right\}, \text { where } \Lambda_{0}=\Lambda\left(\omega_{1}(n)\right) .
$$

Let $L_{j}$ denote the length of the longest path through the ApN defined by $T_{j}$ and the $N_{j}$ vertices it contains, $1 \leq j \leq \tau$. For the remainder of the section let

$$
\omega_{0}=\omega_{1}\left(\Lambda_{0}\right), \quad \phi_{0}=\phi\left(\Lambda_{0}, \omega_{0}\right)=\exp \left\{\frac{\omega_{0}}{2 \log \omega_{0}}\right\}, \quad \omega_{2}=\frac{\phi_{0}}{\omega_{0}} .
$$

Then let

$$
J_{1}=\left\{j \in J: L_{j} \geq \frac{N_{j}}{\omega_{1}\left(N_{j}\right)}\right\}
$$

We note that

$$
\begin{aligned}
\log \omega_{2} & =\log \phi_{0}-\log \omega_{0}=\frac{\log \Lambda_{0}}{\omega_{0} \log \log \Lambda}-\log \omega_{0} \\
& =\frac{\omega_{0}^{2}}{(2+o(1)) \omega_{0} \log \log \omega_{0}}-\log \omega_{0} .
\end{aligned}
$$

For $j \in J, N_{j} \geq \Lambda_{0}$ (see (5.6)). It follows from Lemma 5.1 that the size of $J_{1}$ is stochastically dominated by $\operatorname{Bin}\left(\tau, 1 / \phi_{0}\right)$. Using the bound

$$
\operatorname{Pr}(\operatorname{Bin}(n, p) \geq \alpha n p) \leq\left(\frac{e}{\alpha}\right)^{\alpha n p}
$$

we find that

$$
\operatorname{Pr}\left(\left|J_{1}\right| \geq \frac{\omega_{2} \tau}{\phi_{0}}\right) \leq\left(\frac{e}{\omega_{2}}\right)^{\omega_{2} \tau / \phi_{0}}
$$

Using this we prove

Lemma 5.2. Suppose that

$$
\log \left(\frac{\sigma}{\tau}\right) \leq \frac{\omega_{0}}{\log \omega_{0}}
$$


Then q.s., for all $\lambda_{1} \leq \tau \ll \sigma \ll N$ and all collections $\mathcal{T}$ of $\tau$ faces of $\mathcal{A}_{\sigma}$ we find that with $J_{1}$ as defined in (5.8),

$$
\left|J_{1}\right| \leq \frac{\omega_{2} \tau}{\phi_{0}}
$$

Proof. It follows from (5.9) that

$$
\begin{aligned}
\operatorname{Pr} & \left(\exists \tau, \sigma, N, \mathcal{T}:\left|J_{1}\right| \geq \frac{\omega_{2}}{\tau \phi_{0}}\right) \\
& \leq n^{3}\left(\begin{array}{c}
(2 \sigma+1) \\
\tau
\end{array}\right)\left(\frac{e}{\omega_{2}}\right)^{\omega_{2} \tau / \phi_{0}} \\
& \leq n^{3}\left(\frac{e(2 \sigma+1)}{\tau} \cdot\left(\frac{e}{\omega_{2}}\right)^{\omega_{2} / \phi_{0}}\right)^{\tau} \\
& \leq \exp \left\{\tau\left(\frac{3 \log n}{\tau}+2+\log \left(\frac{\sigma}{\tau}\right)+\frac{\omega_{2}}{\phi_{0}}-\frac{\omega_{2} \log \omega_{2}}{\phi_{0}}\right)\right\} \\
& \leq \exp \left\{\tau\left(\frac{3 \log n}{\tau}+2+\frac{\omega_{0}}{\log \omega_{0}}+-\frac{\omega_{0}}{(2+o(1)) \log \log \omega_{0}}\right)\right\} \\
& =O\left(n^{- \text {anyconstant }}\right) .
\end{aligned}
$$

\section{PROOF OF THEOREM 1.2}

Fix a path $P$ of $\mathcal{A}_{n}$. Suppose that, after adding $\sigma \geq n^{1 / 2}$ vertices, we find that $P$ visits

$$
n^{1 / 2} \geq \tau \geq \lambda_{1} \omega_{0}
$$

of the triangles $T_{1}, T_{2}, \ldots, T_{\tau}$ of $\mathcal{A}_{\sigma}$. Now consider adding $N$ more vertices, where the value of $N$ is given in (6.4) below. Let $\sigma^{\prime}=\sigma+N$ and let $\tau^{\prime}$ be the number of triangles of $\mathcal{A}_{\sigma^{\prime}}$ that are visited by $P$.

We assume that

$$
\frac{\alpha}{2} \log \log n \leq \log \left(\frac{\sigma}{\tau}\right) \leq \frac{\omega_{0}}{\log \omega_{0}} .
$$

Let $M_{i}$ be the number of vertices placed in $T_{i}$ and let $N_{i}$ be the number of these that are visited by $P$. It follows from Lemma 4.1 that w.h.p.

$$
\sum_{i=1}^{\tau} M_{i} \leq \frac{100 \tau N}{\sigma} \log \left(\frac{\sigma}{\tau}\right)
$$


Now w.h.p.,

$$
\sum_{i=1}^{\tau} N_{i} \leq \tau \Lambda_{0}+\frac{100 \omega_{2} \tau N}{\phi_{0} \sigma} \log \left(\frac{\sigma \phi_{0}}{\omega_{2} \tau}\right)+\frac{100 \tau N}{\sigma \omega_{0}} \log \left(\frac{\sigma}{\tau}\right) .
$$

To see (6.3), observe that $\tau \Lambda_{0}$ bounds the contribution from $[\tau] \backslash J$ (see (5.6)). The second term bounds the contribution from $J_{1}$. Now $\left|J_{1}\right|<\omega_{2} \tau / \phi_{0} \ll \tau$ as shown in Lemma 5.2. We cannot apply Lemma 4.1 to bound the contribution of $J_{1}$ unless we know that $\left|J_{1}\right| \geq \lambda_{1}$. We choose an arbitrary set of indices $J_{2} \subseteq[\tau] \backslash J_{1}$ of size $\omega_{2} \tau / \phi_{0}-\left|J_{1}\right|$ and then the middle term bounds the contribution of $J_{1} \cup J_{2}$. Note that $\omega_{2} \tau / \phi_{0}=\tau / \omega_{0} \geq \lambda_{1}$ from (6.1). The third term bounds the contribution from $J \backslash J_{1}$. Here, we use $\omega_{1}\left(N_{j}\right) \geq \omega_{1}\left(\Lambda_{0}\right)=\omega_{0}$; see (5.8).

We now choose

$$
N=3 \sigma \Lambda_{0}
$$

We observe that

$$
\begin{gathered}
\frac{\omega_{2}}{\phi_{0}} \log \left(\frac{\sigma \phi_{0}}{\omega_{2} \tau}\right) \leq \frac{1}{\omega_{0}}\left(\frac{\omega_{0}}{\log \omega_{0}}+2 \log \omega_{0}\right)=o(1) . \\
\frac{1}{\omega_{0}} \log \left(\frac{\sigma}{\tau}\right) \leq \frac{1}{\log \omega_{0}}=o(1) .
\end{gathered}
$$

Now, along with Lemma 3.1, this implies that

$$
\tau^{\prime} \leq \sum_{i=1}^{\tau}\left(N_{i}+1\right) \leq \tau+\tau \Lambda_{0}+o\left(\frac{\tau N}{\sigma}\right) .
$$

Since $\sigma^{\prime}=\sigma+N$, this implies that

$$
\frac{\tau^{\prime}}{\sigma^{\prime}} \leq\left(\frac{1}{3}+o(1)\right) \frac{\tau}{\sigma}<\frac{\tau}{2 \sigma} .
$$

It follows by repeated application of this argument that we can replace Theorem 1.1 by the following lemma:

\section{Lemma 6.1.}

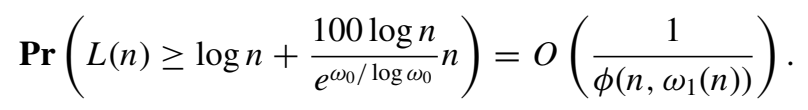

Proof. We add the vertices in rounds of size $\sigma_{0}=n^{1 / 2}, \sigma_{1}, \ldots, \sigma_{m}$. Here, $\sigma_{i}=3 \sigma_{i-1} \Lambda_{0}$ and $m-1 \geq(1-o(1)) \frac{\log n}{\log \Lambda_{0}}=(1-o(1)) \frac{\log n}{\omega_{1}(n)^{2}}=\log ^{1-2 \alpha} n$. We let $P_{0}, P_{1}, P_{2}, \ldots, P_{m}=P$ be a sequence of paths, where $P_{i}$ is a path in $\mathcal{A}_{i}=\mathcal{A}_{\sigma_{0}+\cdots+\sigma_{i}}$. Furthermore, $P_{i}$ is obtained 
from $P_{i+1}$ in the same way that $Q$ is obtained from $P$ in Lemma 3.1. We let $\tau_{i}$ denote the number of faces of $\mathcal{A}_{i}$ whose interior is visited by $P_{i}$. It follows from Lemma 3.1 and Lemma 4.1 that the length of $P$ is bounded by

$$
m+\frac{\tau_{m-1}}{\sigma_{m-1}} \sigma_{m} \log \left(\frac{\sigma_{m-1}}{\tau_{m-1}}\right),
$$

since the second term is a bound on the number of points in the interior of triangles of $\mathcal{A}_{m-1}$ visited by $P$.

We have w.h.p. that

$$
\frac{\sigma_{i}}{\tau_{i}} \geq\left\{\begin{array}{ll}
\frac{2 \sigma_{i-1}}{\tau_{i-1}} & \frac{\sigma_{i-1}}{\tau_{i-1}} \leq e^{\omega_{0} / \log \omega_{0}} \\
\frac{\sigma_{i-1}}{100 \tau_{i-1} \log \left(\sigma_{i-1} / \tau_{i-1}\right)} & \frac{\sigma_{i-1}}{\tau_{i-1}}>e^{\omega_{0} / \log \omega_{0}}
\end{array} .\right.
$$

The second inequality here is from Lemma 4.1.

The result follows from $2^{\log ^{1-2 \alpha} n} \geq e^{\omega_{0} / \log \omega_{0}}$.

To get Theorem 1.2, we repeat the argument in Sections 5 and 6, but we start with $\omega_{1}(x)=\log ^{1 / 3} x$. The claim in Theorem 1.2 is then slightly weaker than the claim in Lemma 6.1.

We note that subsequent to completion of this paper, Theorem 1.2 has been improved [2] with a high probability upper bound of $O\left(n^{1-\varepsilon}\right)$ on $L(n)$.

\section{FUNDING}

Supported in part by EPSRC grant EP/J006300/1. Supported in part by NSF grant CCF0502793.

\section{REFERENCES}

[1] G. Chen and X. Yu. "Long Cycles in 3-Connected Graphs." Journal of Combinatorial Theory B 86 (2002), 80-99.

[2] A. Collevecchio, A. Mehrabian, and N. Wormald. "Longest Paths in Random Apollonian Networks and Largest $r$-ary Subtrees of Random $d$-ary Recursive Trees." Cornell University Library. Available online (arxiv.org/abs/1404.2425), 2014.

[3] C. Cooper and A. M. Frieze. "The Height of Random $k$-Trees and Related Branching Processes." Cornell University Library. Available online (arxiv.org/abs/1309.4342), 2013.

[4] E. Ebrahimzadeh, L. Farczadi, P. Gao, A. Mehrabian, C. Sato, N. Wormald, and J. Zung. "On the Longest Paths and the Diameter in Random Apollonian Networks." Available online (arxiv.org/pdf/1303.5213v1.pdf), 2013.

[5] A. M. Frieze and C. Tsourakakis. "On Certain Properties of Random Apollonian Networks." WAW 2012 (2012), 93-112. 